\title{
The contributions of biosynthesis and acyl chain remodelling to the molecular species profile of phosphatidylcholine in yeast
}

\author{
H.A. Boumann' and A.I.P.M. de Kroon ${ }^{2}$
}

Department Biochemistry of Membranes, Bijvoet Institute and Institute of Biomembranes, Utrecht University, Padualaan 8, 3584 CH Utrecht, The Netherlands

\begin{abstract}
Phosphatidylcholine $(\mathrm{PC})$ is a very abundant membrane lipid in most eukaryotes, including yeast. The molecular species profile of $\mathrm{PC}$, i.e. the ensemble of $\mathrm{PC}$ molecules with acyl chains differing in number of carbon atoms and double bonds, is important for membrane function. Pathways of PC synthesis and turnover maintain PC homoeostasis and determine the molecular species profile of PC. Studies addressing the processes involved in establishing the molecular species composition of $\mathrm{PC}$ in yeast using stable isotope labelling combined with detection by MS are reviewed.
\end{abstract}

\section{Introduction}

It is widely recognized that the glycerophospholipid composition of biological membranes is important for their function. Both the phospholipid class composition, defined by the nature of the polar head group, and the composition of the hydrocarbon chains attached to the glycerol backbone determine the physical properties of a biomembrane, and are subject to adaptations in response to a changing environment in many organisms. These physical properties include membrane thickness, intrinsic curvature and fluidity, which affect key functions such as the membrane barrier function, the activity of membrane-associated enzymes, and processes like membrane fusion and fission. In view of the sheer abundance of PC (phosphatidylcholine) in eukaryotic membranes - it often constitutes $>50 \%$ of total membrane phospholipids [1] - the acyl chain composition of PC is paramount for proper membrane function. As the major bilayer-forming phospholipid, PC plays an important role in preserving the membranes' barrier function. In addition, PC plays an important role in signal transduction as a source of lipid signalling molecules, such as lyso-PC, phosphatidic acid and DAG (diacylglycerol) [2,3].

The yeast Saccharomyces cerevisiae is an excellent model organism to elucidate the mechanisms of the establishment and maintenance of the PC acyl chain composition, because of its easily accessible molecular biology, and also because its glycerophospholipid biosynthetic machinery strongly resembles that of mammalian cells. Additional advantages of yeast include its relatively simple repertoire of acyl chains, with palmitic $\left(\mathrm{C}_{16: 0}\right)$, palmitoleic $\left(\mathrm{C}_{16: 1}\right)$, stearic $\left(\mathrm{C}_{18: 0}\right)$ and

Key words: acyl chain composition, deacylation, electrospray ionization tandem MS (ESIMS/MS), phosphatidylcholine, reacylation, yeast.

Abbreviations used: CID, collision-induced dissociation; DAG, diacylglycerol; ESI-MS/MS, electrospray ionization tandem MS; PC, phosphatidylcholine; PE, phosphatidylethanolamine.

${ }^{1}$ Present address: Royal Netherlands Institute for Sea Research, Department of Marine Biogeochemistry and Toxicology, Den Burg - Texel, The Netherlands.

${ }^{2}$ To whom correspondence should be addressed (email a.i.p.m.dekroon@chem.uu.nl). oleic acid $\left(\mathrm{C}_{18: 1}\right)$ accounting for $95 \%$ of total fatty acids [4], its lack of sphingomyelin and alkyl-linked glycerophospholipids, and the ease of incorporating labelled lipid precursors.

Yeast has two biosynthetic pathways leading to PC that are also found in mammalian cells (Scheme 1). PC is the end-product of the CDP-DAG pathway, in which PS (phosphatidylserine) and PE (phosphatidylethanolamine) are important membrane lipid intermediates [5]. In the final steps, $\mathrm{PE}$ is methylated to PC by the phospholipid- $N$-methyltransferases Cho2 and Opi3 localized in the endoplasmic reticulum [6]. Using $S$-adenosyl-methionine as the methyl donor, Cho2 methylates the phosphoethanolamine headgroup of PE yielding phosphatidylmonomethylethanolamine (PMME), which is subsequently converted into PC via phosphatidyldimethylethanolamine (PDME) by Opi3 [7-9]. In the complementary CDP-choline pathway, choline is phosphorylated by the kinase Cki1 to phosphocholine, which is converted into CDP-choline by the CTP-phosphocholine cytidylyltransferase Pct1. Finally, the choline phosphotransferase Cpt1p transfers CDP-choline to DAG to yield PC [5]. Ept1, the corresponding enzyme from the CDP-ethanolamine pathway, also contributes to PC synthesis, the extent depending on the strain background [10]. The CDP-choline pathway only contributes to net PC synthesis if choline is present in the culture medium. In its absence, this pathway serves to recycle choline from PC turnover [11].

Whereas the biosynthesis of PC has been extensively studied, knowledge on PC metabolism downstream of synthesis is scarce. To date, the in vivo metabolic conversions documented at the gene level (Scheme 1) are limited to the turnover of PC by the SPO14-encoded phospholipase D that is essential for sporulation [12], the deacylation of PC by Plb1p [13], and the temperature-dependent and cholineinduced conversion of PC into glycerophosphocholine by Nte1 that is specific for CDP-choline-derived PC $[14,15]$. Remodelling of PC by acyl chain exchange was inferred from 


\section{Scheme $1 \mid$ Metabolism of PC in yeast}

The biosynthetic routes leading to $P C$ and the conversions of $P C$ detected in vivo and documented at the gene level are depicted. The enzymes catalysing the individual steps are indicated. PDME, phosphatidyldimethylethanolamine; PMME, phosphatidylmonomethylethanolamine.

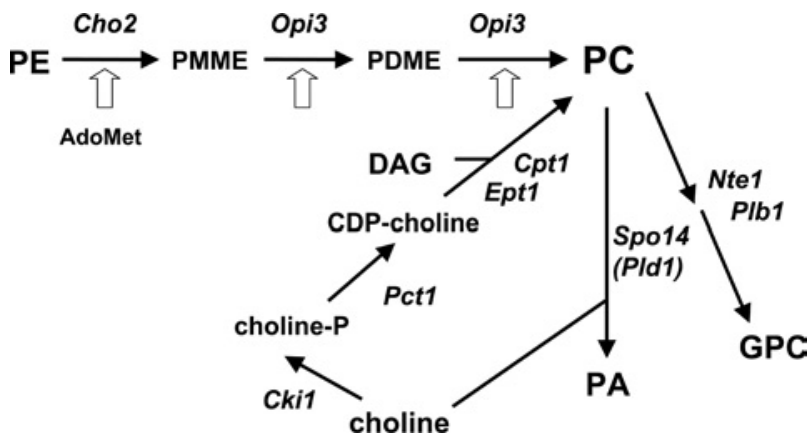

the selective incorporation of exogenous radiolabelled acyl chains at the $s n-2$ position of PC [4].

In this paper, our current understanding of the processes involved in the establishment of the PC molecular species profile is summarized.

\section{ESI-MS/MS (electrospray ionization tandem MS) analysis of PC molecular species profiles}

Today, MS is the most sensitive method for a detailed structural characterization of biological molecules including phospholipids [16]. The major advantages of this technique include: (i) its sensitivity as only pmol amounts of samples are required, (ii) the use of unfractionated total lipid extracts for direct analysis of different phospholipid classes, (iii) the identification of individual molecular species, (iv) the ability to quantify data and (v) the possibility to perform metabolic labelling studies.

ESI-MS/MS has proven to be an excellent technique to perform full analysis of complex lipid mixtures $[17,18]$. The conventional instrument applied for MS/MS experiments is the triple quadrupole instrument, in which the first (MS1) and third (MS2) quadrupoles are identical and operate as mass analysers, while the second quadrupole serves as a collision cell. The introduction of an inert gas, e.g. argon, in the collision cell leads to the decomposition of incoming ions in a process referred to as CID (collision-induced dissociation). In most cases, phospholipids are analysed by using low-energy CID to specifically cleave off the head group moiety [17]. For quantitative analysis of PC species compositions, parent ion scanning in the positive ion mode is the method of choice. In this mode, the MS1 analyser consecutively scans a selected $\mathrm{m} / \mathrm{z}$ range. Ions entering the collision cell will be subjected to CID, in which PC molecular species yield the characteristic phosphocholine moiety with an $m / z$ of 184 . The MS2 analyser is set to transfer only the preselected fragments that are typical for the phospholipid of interest. In the case of PC, recording
Figure 1 | Steady-state PC species profiles obtained by parent ion scanning for $m / z 184$ of wild-type (BY4742), pct1 and cho2opi3 cells cultured to mid-exponential phase in semi-synthetic lactate medium

The culture medium of the chozopi3 strain was supplemented with $1 \mathrm{mM}$ choline. ESI-MS/MS data were obtained and quantified as described in $[19,26]$.

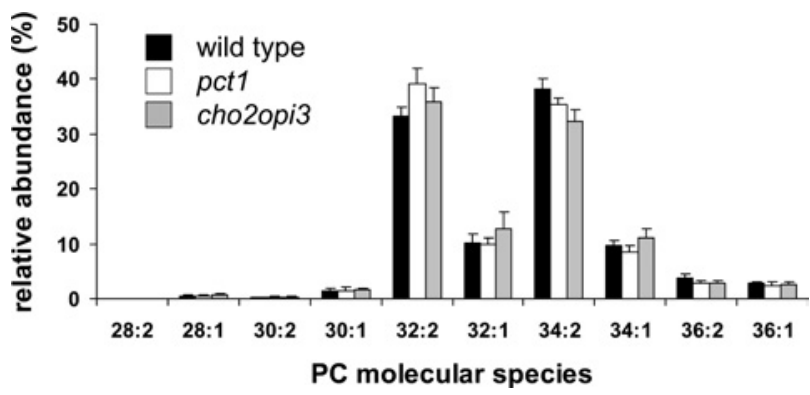

proceeds if the parental ions yield a fragment with an $\mathrm{m} / \mathrm{z}$ value of 184 .

Parent ion scanning for $\mathrm{m} / \mathrm{z} 184$ of total lipid extracts of wild-type yeast cells (BY4742) grown to mid-exponential phase revealed a PC species profile (quantified in Figure 1) containing four prominent components: diunsaturated 32:2 (containing two $\mathrm{C}_{16: 1}$ acyl chains) and 34:2 (containing a $\mathrm{C}_{16: 1}$ and $\mathrm{C}_{18: 1}$ acyl chain), and the mono-unsaturated species 32:1 (composed of $\mathrm{C}_{16: 0}$ and $\mathrm{C}_{16: 1}$ ) and 34:1 (composed of $\mathrm{C}_{16: 0}$ and $\mathrm{C}_{18: 1}$ and/or of $\mathrm{C}_{18: 0}$ and $\mathrm{C}_{16: 1}$ ) [19]. Please note that except for 32:2, each of these components may correspond to two (32:1 and 34:2) or four (34:1) molecular species due to positional isomers, of which the relative amounts were not determined. The acyl chain composition of yeast cells strongly depends on the carbon source used to culture the cells [20]. Because of our interest in phospholipid metabolism under conditions of full mitochondrial development, lactate was used as carbon source unless indicated otherwise.

\section{Species selectivity of the PC biosynthetic routes}

To get a first insight into the possibility that the two PC biosynthetic pathways contribute differently at the level of molecular species, the steady-state PC species profile of wildtype cells was compared with those of cho2opi3 and pct1 mutants lacking the PE methylation and the CDP-choline route respectively (Figure 1). The three profiles are similar (in agreement with [14]), suggesting that either the contributions of the two routes are similar or that other processes compensate for differences in contribution.

Pulse labelling of cells with stable isotope-labelled precursors and subsequent analysis of phospholipid molecular species by ESI-MS/MS allows distinction between newly synthesized and pre-existing pools of most phospholipid classes, based on the difference in molecular mass conferred by the isotope labels (see e.g. [21]). To distinguish the contributions of the two PC biosynthetic routes to the PC 
Figure 2 | Profiles of the newly synthesized PC species obtained via $\mathrm{PE}$ methylation and the CDP-choline pathway compared with the steady-state $\mathrm{PC}$ species distribution

Wild-type cells were pulsed with $\left[\right.$ methyl- $\left.{ }^{2} \mathrm{H}_{3}\right]$ methionine and $\left[{ }^{2} \mathrm{H}_{13}\right]$ choline for 10 min. Subsequently, newly synthesized PC was detected by parent-ion scanning of total lipid extracts for m/z 193 and 197 respectively. The steady-state PC species profile was obtained in parent ion scans for $m / z$ 184. Data were obtained and quantified as described in [19].

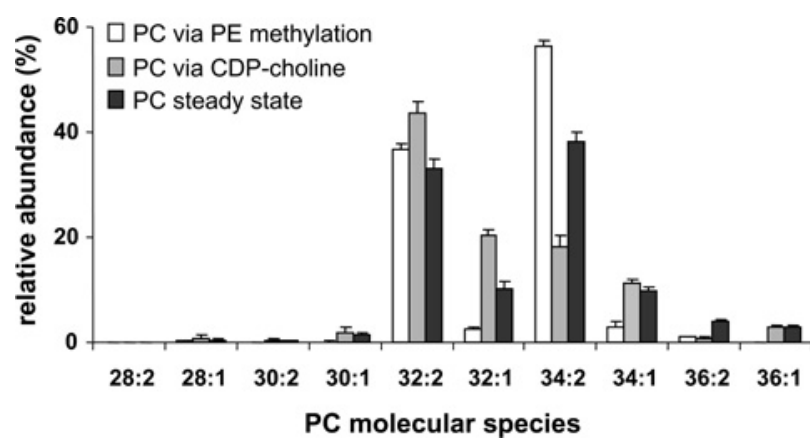

species profile, wild-type yeast was pulsed for $10 \mathrm{~min}$ with ${ }^{2} \mathrm{H}$-labelled substrates. The PE methylation pathway was addressed by pulse labelling with $\left[\right.$ methyl $\left.-{ }^{2} \mathrm{H}_{3}\right]$ methionine yielding $\left[\text { methyl }-{ }^{2} \mathrm{H}_{3}\right]_{3} \mathrm{PC}$ that is selectively visualized by ESI-MS/MS in a parent ion scan for $m / z$ 193. Upon incubation with perdeuterated $\left[{ }^{2} \mathrm{H}_{13}\right]$ choline, the CDP-choline route produces $\left[{ }^{2} \mathrm{H}_{13}\right] \mathrm{PC}$ that is detected by parent ion scanning for $m / z$ 197. As shown in Figure 2, the methylation of PE and the CDP-choline route produce the PC species in different ratios. Whereas $\mathrm{PE}$ methylation predominantly yields diunsaturated PC, the CDP-choline route produces a more diverse profile of PC species (Figure 2). Moreover, the profiles of newly synthesized PC differ from that of steadystate PC [19].

The species profile of newly methylated PC was compared with that of its precursor PE that was determined by neutral loss scanning in the positive-ion mode for $\mathrm{m} / \mathrm{z} 141$. In this scanning mode, the scans by the MS1 and MS2 analysers are synchronized with a fixed $\mathrm{m} / \mathrm{z}$ difference equivalent to the selected neutral loss fragment. Upon CID, PE ions lose the phosphoethanolamine moiety as an uncharged fragment of $141 \mathrm{Da}$. The recoding device registers only those ions that have lost the fragment of interest. Compared with the species profile of cellular PE that is dominated by 32:2 PE ( $\sim 20 \%)$ and $34: 2 \mathrm{PE}(\sim 65 \%)$, the derived newly synthesized $\mathrm{PC}$ is enriched in the $32: 2$ species ( $\sim 35 \%$, Figure 2$)$, suggesting that the methyltransferases preferentially convert 32:2 PE. When the species composition of the PE precursor pool was varied, by using different carbon sources or a phospholipid biosynthetic mutant strain, a similar enrichment was observed [22].

To obtain further insight into the apparent preference for methylating 32:2 PE, the reaction was investigated in vitro, by incubating isolated microsomes for $10 \mathrm{~min}$ with $S$-[metbyl${ }^{2} \mathrm{H}_{3}$ ] adenosyl-methionine. Again, a similar preferential con- version of 32:2 PE into PC was detected, indicating that it is intrinsic to the methyltransferases in microsomes, and not dependent on the intermembrane transport processes required to supply the enzymes with $\mathrm{PE}$ that is for the most part synthesized by mitochondrial Psd1 and by Psd2 in the Golgi/vacuole [23,24]. Consistent with this conclusion, a recent analysis of the species distributions of the aminoglycerophospholipids in yeast subcellular fractions did not reveal species selectivity in interorganelle phospholipid transport [25]. The preferential methylation of 32:2 $\mathrm{PE}$ is for the most part conferred by $\mathrm{Cho} 2$ with a minor contribution of Opi3 [22].

The contributions of Cpt1 and Ept1 to CDP-choline derived $\mathrm{PC}$ were examined by pulsing ept 1 and $c p t 1$ cells with $\left[{ }^{2} \mathrm{H}_{13}\right]$ choline respectively. The species profile of newly synthesized PC in the ept1 strain was virtually identical with that in wild-type cells, and differed significantly from that obtained in the cpt1 strain in which 34:2 PC was the major species [26]. From this result, it was concluded that in the BY4742 strain background, Ept1 does not contribute substantially to PC biosynthesis (cf. $[10,11]$ ).

\section{Acyl chain remodelling of PC}

Are the contributions of PE methylation and the CDPcholine pathway sufficient to account for the steady-state PC species distribution in wild-type cells? Inspection of Figure 2 reveals that the steady-state PC species profile cannot be fitted to any weighted average of the two profiles of newly synthesized PC, implicating other processes in addition to the biosynthetic routes in establishing the steadystate distribution. The PC acyl chain composition could be modified by species-selective degradation, and/or by acyl chain remodelling, i.e. exchange of acyl chains. In the pct1 and cho2opi3 mutants, lacking either of the pathways (Figure 1), the need for processing of newly synthesized PC to accomplish the steady-state profile is even more evident $[19,26]$. The post-synthetic processing of the newly synthesized PC profiles towards the steady state was visualized in these strains in prolonged labelling and pulse-chase experiments with $\left[\right.$ methyl $\left.-{ }^{2} \mathrm{H}_{3}\right]$ methionine [19] and $\left[{ }^{2} \mathrm{H}_{13}\right]-$ choline (H.A. Boumann, unpublished work) respectively, and proceeds with an apparent half-time in the order of $1 \mathrm{~h}$.

In the pct1 strain, a species profile containing relatively small proportions of newly synthesized 32:1 and 34:1 PC evolves to a steady-state profile containing at least twice the initial amounts of these species [19]. Species-selective degradation alone is unlikely to account for this process, as it would involve degradation of some $50 \%$ of newly synthesized PC. Moreover, during prolonged labelling with deuterated PC precursors, no change in species composition of the pre-existing PC pool was observed in wild-type cells, arguing against the occurrence of extensive species-selective turnover. This leaves remodelling by acyl chain exchange as the more plausible mechanism. ESI-MS/MS analysis of lyso-PC, after treatment of the pct1 lipid extracts with phospholipase $A_{2}$, revealed the substitution of $C_{16: 1}$ acyl chains 
esterified at the $s n-1$ position of newly synthesized PC by other acyl chains, unequivocally demonstrating the occurrence of acyl chain remodelling [19].

\section{Conclusion}

The PC species profile in wild-type yeast is to a large extent determined by the species selectivity of the methyltransferases converting PE into PC. The CDP-choline pathway using exogenous choline or choline from PC turnover adds to the profile by producing the major PC species in a different characteristic ratio. However, the relative contributions of the two pathways to the total PC pool are unknown and depend on the culture conditions. Stable isotope labelling combined with detection by ESI-MS/MS has revealed that, irrespective of their relative contributions, the two biosynthetic pathways are insufficient to account for the steadystate PC species profile. Acyl-chain remodelling and possibly species-selective degradation are also involved, and can be conveniently studied in mutants lacking one of the biosynthetic pathways. Remodelling of newly synthesized PC by acyl chain exchange was demonstrated in the pct 1 mutant.

Remodelling of phospholipids is generally thought to proceed via deacylation to a lyso-phospholipid mediated by a phospholipase A or B, followed by an acyl-CoA-dependent reacylation by an acyltransferase [27]. Alternatively, acyl chain shuffling via transacylation may occur in which another phospholipid serves as acyl chain donor and a lyso-phospholipid as the acceptor (see e.g. [28]). Both acyl-CoAdependent and acyl-CoA-independent acylation of lyso-PC have been reported in yeast [29,30]. Our current research is directed at identifying the genes involved in shaping the PC species profile in order to elucidate the biological functions of PC remodelling.

We gratefully acknowledge Professor Dr Albert Heck (Utrecht University) for sharing mass spectrometric facilities and expertise and Professor Dr Ben de Kruijff (Utrecht University) and the yeast lipid group in the Department of Biochemistry of Membranes for fruitful discussions. This work was supported by the Netherlands Division of Chemical Sciences with financial aid from the Netherlands Organization for Scientific Research.

\section{References}

1 Kent, C. and Carman, G.M. (1999) Trends Biochem. Sci. 24, 146-150

2 Exton, J.H. (1994) Biochim. Biophys. Acta 1212, 26-42

3 Cui, Z. and Houweling, M. (2002) Biochim. Biophys. Acta 1585 87-96

4 Wagner, S. and Paltauf, F. (1994) Yeast 10, 1429-1437

5 Carman, G.M. and Henry, S.A. (1999) Prog. Lipid Res. 38, 361-399

6 Zinser, E., Sperka-Gottlieb, C.D., Fasch, E.V., Kohlwein, S.D., Paltauf, F. and Daum, G. (1991) J. Bacteriol. 173, 2026-2034

7 Kodaki, T. and Yamashita, S. (1987) J. Biol. Chem. 262, 15428-15435

8 Summers, E.F., Letts, V.A., McGraw, P. and Henry, S.A. (1988) Genetics 120, 909-922

9 McGraw, P. and Henry, S.A. (1989) Genetics 122, 317-330

10 Henneberry, A.L., Lagace, T.A., Ridgway, N.D. and McMaster, C.R. (2001) Mol. Biol. Cell 12, 511-520

11 McMaster, C.R. and Bell, R.M. (1994) J. Biol. Chem. 269, 28010-28016

12 Rudge, S.A. and Engebrecht, J. (1999) Biochim. Biophys. Acta 1439 167-174

13 Lee, K.S., Patton, J.L., Fido, M., Hines, L.K., Kohlwein, S.D., Paltauf, F., Henry, S.A. and Levin, D.E. (1994) J. Biol. Chem. 269, 19725-19730

14 Dowd, S.R., Bier, M.E. and Patton-Vogt, J.L. (2001) J. Biol. Chem. 276 3756-3763

15 Zaccheo, 0., Dinsdale, D., Meacock, P.A. and Glynn, P. (2004) J. Biol. Chem. 279, 24024-24033

16 Han, X. and Gross, R.W. (2003) J. Lipid Res. 44, 1071-1079

17 Brügger, B., Erben, G., Sandhoff, R., Wieland, F.T. and Lehmann, W.D. (1997) Proc. Natl. Acad. Sci. U.S.A. 94, 2339-2344

18 Schneiter, R., Brügger, B., Sandhoff, R., Zellnig, G., Leber, A., Lampl, M. Athenstaedt, K., Hrastnik, C., Eder, S., Daum, G. et al. (1999) J. Cell Biol. 146, 741-754

19 Boumann, H.A., Damen, M.J., Versluis, C., Heck, A.J., de Kruijff, B. and de Kroon, A.I. (2003) Biochemistry 42, 3054-3059

20 Tuller, G., Nemec, T., Hrastnik, C. and Daum, G. (1999) Yeast 15, 1555-1564

21 DeLong, C.J., Shen, Y.J., Thomas, M.J. and Cui, Z. (1999) J. Biol. Chem. 274, 29683-29688

22 Boumann, H.A., Chin, P.T., Heck, A.J., De Kruijff, B. and De Kroon, A.I. (2004) J. Biol. Chem. 279, 40314-40319

23 Trotter, P.J. and Voelker, D.R. (1995) J. Biol. Chem. 270, 6062-6070

24 Birner, R., Burgermeister, M., Schneiter, R. and Daum, G. (2001) Mol. Biol. Cell 12, 997-1007

25 Bürgermeister, M., Birner-Grünberger, R., Heyn, M. and Daum, G. (2004) Biochim. Biophys. Acta 1686, 148-160

26 Boumann, H.A., De Kruijff, B., Heck, A.J. and De Kroon, A.I. (2004) FEBS Lett. 569, 173-177

27 Yamashita, A., Sugiura, T. and Waku, K. (1997) J. Biochem. (Tokyo) 122, 1-16

28 Yamashita, A., Watanabe, M., Sato, K., Miyashita, T., Nagatsuka, T., Kondo, H., Kawagishi, N., Nakanishi, H., Kamata, R., Sugiura, T. et al. (2003) J. Biol. Chem. 278, 30382-30393

29 Richard, M.G. and McMaster, C.R. (1998) Lipids 33, 1229-1234

30 Testet, E., Laroche-Traineau, J., Noubhani, A., Coulon, D., Bunoust, O., Camougrand, N., Manon, S., Lessire, R. and Bessoule, J.). (2005) Biochem. J. 387, 617-626

Received 21 June 2005 